Gut, 1978, 19, 685-688

\title{
Computed tomography, ultrasound, and cholestatic jaundice
}

\author{
A. I. MORRIS ${ }^{1}$, R. A. FAWCITT, R. WOOD, W. S. C. FORBES, \\ I. ISHERWOOD, AND M. N. MARSH
}

From the University Department of Medicine and Department of Radiology, Hope Hospital (University of Manchester School of Medicine), Salford, and the University Department of Diagnostic Radiology, The Medical School, Manchester

SUMMARY Computed tomography detected the presence of dilated ducts in 15 of 18 cases with extrahepatic obstructive jaundice and demonstrated the obstructing lesion in 18 out of 25 . The cause of the obstruction was detected in the absence of duct dilatation, and calculi which were radiolucent on conventional radiographs were demonstrated. Ultrasound detected dilated ducts in eight of 10 cases, but demonstrated the cause of the obstruction in only one of 13 cases. Both techniques are capable of demonstrating dilated ducts in the majority of cases, but computed tomography is better at detecting the cause of the obstruction.

The differentiation between extrahepatic biliary obstruction and other causes of jaundice is often difficult, yet clearly important in patient management.

The introduction of the Chiba needle for percutaneous transhepatic cholangiography has provided an accurate method of delineating the biliary tract in patients with both extra and intrahepatic cholestasis. This invasive technique has, however, a recognised morbidity (Elias, 1976).

A variety of non-invasive methods have been suggested and the method currently most widely used is ultrasound. In expert hands, this technique can detect most dilated bile ducts, but is generally less successful in demonstrating the obstructing lesion (Vicary et al., 1977). The technique is particularly dependent on operator skill and experience.

The development of whole body computed tomography has provided a further non-invasive method. Preliminary reports from Great Britain (Fawcitt et al., 1978; Kreel, 1977; Morris et al., 1977) and reports from the United States (Havrilla et al., 1977; Stephens et al., 1977) have suggested its value in the diagnosis of liver disease. In this paper we describe our experience with computed tomography and

\footnotetext{
${ }^{1}$ Address for reprints: Dr A. I. Morris, Department of Medicine, Hope Hospital, Eccles Old Road, Salford, M6 8HD.

Received for publication 13 January 1978
}

compare it with greyscale ultrasonography in the diagnosis of obstructive jaundice.

\section{Methods}

PATIENTS

Forty-one consecutive patients with cholestatic jaundice presenting to the University Department of Medicine were referred for computed tomography, and 20 of them were also examined by ultrasonud. Those patients who underwent both investigations had them performed within one week of each other.

All diagnoses were proven by Chiba needle percutaneous transhepatic cholangiography (PTC), laparotomy, liver biopsy, or a combination of these techniques. The presence or absence of dilated bile ducts, in all the patients with extrahepatic biliary obstruction, was confirmed either by PTC or operative cholangiography at the time of laparotomy.

Computed tomography scan examinations were carried out with an EMI CT5000 or CT5005 whole body scanner. Preparation of the patients included the administration of Isogel for two days before the scan, and intravenous or intramuscular propantheline or Buscopan at the time of examination to reduce bowel gas and motility artefacts. Patients were scanned supine, and on occasion in the right lateral decubitus position after oral gastrografin to aid delineation of the duodenal loop.

Ultrasound examinations using a Nuclear Enterprises, grey scale Ultradiasonograph, and PTC 685 
examinations were performed by one radiologist (R.W.).

\section{Results}

Of the 41 patients, 25 had extrahepatic biliary obstruction, and 16 had intrahepatic cholestasis. The causes of the jaundice in the 41 patients are shown in the Table.

Table Causes of cholestatic jaundice in 41 patients

\begin{tabular}{lc}
\hline & Number \\
\hline Extrahepatic & 14 \\
Carcinoma pancreas/common bile duct & 10 \\
Gallstones & 1 \\
Chronic pancreatitis & 25 \\
Total & \\
Intrahepatic & 7 \\
Drug & 5 \\
Hepatitis & 2 \\
Chronic active hepatitis & 1 \\
Sclerosing cholangitis & 1 \\
Malignant deposits & 16 \\
Total & \\
\hline
\end{tabular}

\section{EXTRAHEPATIC CHOLESTASIS}

There were 25 patients in this group and in 18 of them dilated ducts were demonstrated by cholangiography, the remaining seven patients having a nondilated biliary tree.

\section{DUCT DILATATION}

\section{Computed tomography}

Of the 18 patients known to have dilated ducts computed tomography provided confirmatory evidence in $15(83 \%)$. In the three remaining cases, despite evidence of duct dilatation at PTC, computed tomography scans failed to demonstrate the dilatation. Dilated ducts were seen on computed tomography as a branching pattern of reduced radiodensity distributed through the liver from the hilum (Fig. 1). Seven patients with proven extrahepatic obstruction did not have duct dilatation at cholangiography. There were no false positive computed tomography observations in this group.

\section{Ultrasound}

Thirteen patients with extrahepatic cholestasis were examined both by ultrasound and computed tomography. Ten had evidence of dilated ducts at cholangiography. These dilated ducts were confirmed by ultrasound in eight. In seven of these eight patients, computed tomography had also shown duct dilatation. Thus, in one case ultrasound revealed dilated ducts which computed tomography had failed to

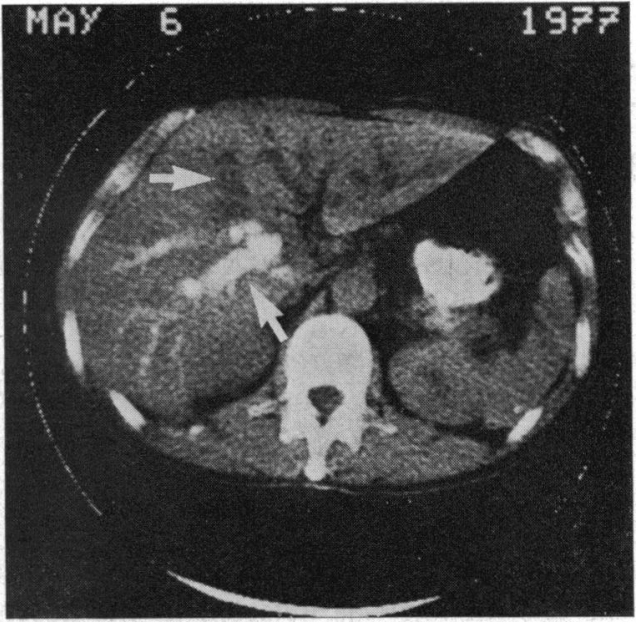

Fig. 1 Dilated intrahepatic bile ducts. Normally seen as low density branching intrahepatic structures $(\rightarrow)$, but computed tomography may show layering of contrast medium in the dependant ducts $(\uparrow)$ following transhepatic cholangiography.

demonstrate. In the two patients where ultrasound had not shown the dilated ducts, however, computed tomography was successful in both cases. There were no false positives with ultrasound, as normal ultrasound scans were reported in the three other patients who had normal ducts at cholangiography.

Using both techniques 16 of the 18 patients with dilated ducts were identified. There were no false positives.

\section{OBSTRUCTING LESION}

\section{Computed tomography}

The cause of obstruction to the biliary tract was correctly diagnosed by computed tomography in 18 of the 25 patients. Fourteen patients had carcinoma of the head of the pancreas or common bile duct and computed tomography demonstrated the tumour in 11 , one of whom had non-dilated ducts. The diagnosis of carcinoma was made when an enlarged, irregular head of pancreas, of non-uniform density was seen (Fig. 2).

Of the 10 patients with extrahepatic cholestasis caused by gallstones, in only one case were the stones seen on routine radiography. Computed tomography, however, demonstrated gallstones in six patients, four of whom did not have dilated ducts.

An example of such a 'radiolucent' stone is shown in Fig. 3, where the calculus can be seen in a dilated common bile duct, within the head of the pancreas. A dilated gallbladder is also shown. In one patient with chronic pancreatitis and a non-dilated biliary tree, 


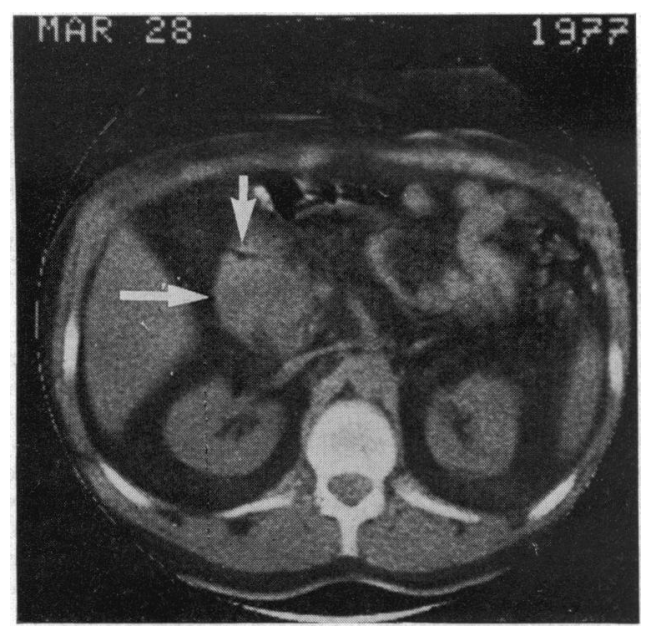

Fig. 2 Carcinoma of head of pancreas. Mass of soft tissue density $(\rightarrow)$ in head of pancreas. A small low density area in the upper margin of the mass $(\downarrow)$ is gas in the deformed duodenal loop.

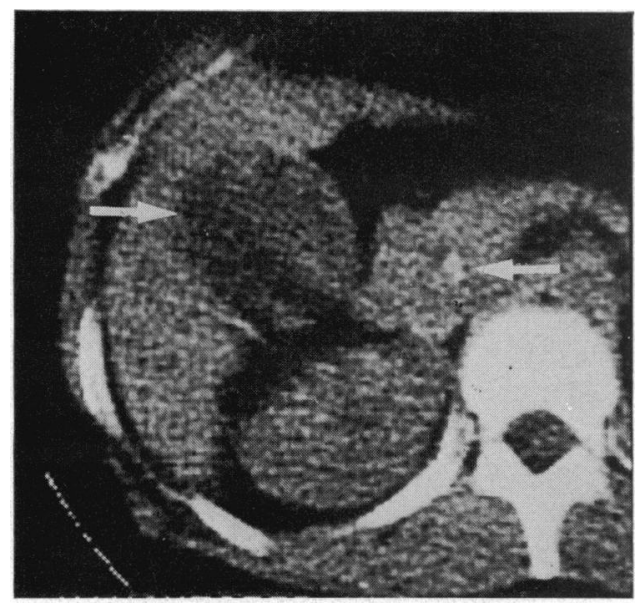

Fig. 3 Gall stone in common bile duct. The conventionally radiolucent calculus $(\leftarrow)$ was impacted in the distal common bile duct, producing dilatation of the biliary tree, including the gall bladder $(\rightarrow)$.

computed tomography demonstrated the enlarged gland, with areas of calcification within it.

\section{Ultrasound}

In only one of the 13 patients examined by ultrasound, a patient with a carcinoma of the head of the pancreas, was the obstructing lesion recognised.

\section{INTRAHEPATIC CHOLESTASIS}

Computed tomography

In only one of the 16 cases of intrahepatic cholestasis were locally dilated 'ducts' reported from the computed tomography scans. These low density areas were subsequently demonstrated to be due to localised metastases. This case represented the only false positive. All other scans were either normal, or showed hepatomegaly, splenomegaly, or hepatosplenomegaly in the patients with hepatitis and chronic active hepatitis.

\section{Ultrasound}

In the seven patients with intrahepatic cholestasis scanned by this technique no abnormality was reported, and in particular no dilated ducts were seen.

\section{Discussion}

Computed tomography has been shown to provide a safe, non-invasive means of detecting dilated ducts in patients with extrahepatic biliary obstruction. The success rate of 15 of 18 patients $(83 \%)$ is very similar to the $88 \%$ quoted by Havrilla et al. (1977). The demonstration of the obstructing lesion in 18 of the 25 cases $(72 \%)$ is again in close agreement with Havrilla ( $77 \%)$. Thus, of 25 patients, duct dilatation or the obstructing lesion was diagnosed in 21 by computed tomography. Of the 16 patients with intrahepatic cholestasis and the seven with extrahepatic obstruction, but without dilated ducts, in only one case were dilated ducts reported on computed tomography (false positive). These were misinterpreted as 'locally' dilated when they were subsequently shown to be due to metastases. The differentiation of metastases from dilated ducts can be difficult (Havrilla et al., 1977) and usually is made on the size, shape, and distribution of the low density areas in adjacent tomographic sections.

The success with which cancer of the head of the pancreas or common bile duct could be identified was encouraging in view of previous reports (Wiggins et al., 1976; Braganza et al., 1977; Fawcitt et al., 1977). This may be due to differing presentations and sites of tumour in other series. All the patients in this series presented with obstructive jaundice, and all but two had large inoperable tumours at laparotomy. The diagnosis of all but four of the cases of gallstones was helpful, particularly as calculi were reported in four patients without duct dilatation.

The success with which ultrasound could demonstrate dilated ducts, in eight of 10 cases, is similar to that reported by Vicary et al. (1977). Our experience suggests that ultrasound is almost as successful as computed tomography in demonstrating dilated ducts, and that using both techniques all of the 10 patients had their dilated ducts identified. Like Vicary et al. we have found ultrasound to be much less successful 
in demonstrating the cause of the obstruction, and the obstructing lesion was identified in only one out of 13 cases.

Computed tomography therefore appears to be more successful in enabling a definitive diagnosis to be made in cases of extrahepatic obstructive jaundice, and the images are more readily recognised. Computed tomography is less dependent on the skill of the operator to produce high quality images.

Ultrasound, however, is more widely available, cheaper, and able to provide a rapid means of detecting dilated ducts.

We wish to thank Professor L. A. Turnberg for his advice and our medical and surgical colleagues for referring their patients.

\section{References}

Braganza, J. M., Fawcitt, R. A., Isherwood, I., Russell, J. G. B., Testa, H. J., Torrance, H. B., and Howat, H. T. (1977). A clinical evaluation of isotope scanning, ultra- sonography and computed tomography in pancreatic disease. Irish Journal of Medical Science, 146 (S1), 27-28.

Elias, E. (1976). Cholangiography in the jaundiced patient. Gut, 17, 801-811.

Fawcitt, R. A., Forbes, W. S. C., Isherwood, I., Braganza, J. M., and Howat, H. T. (1977). Computed tomography in pancreatic disease. British Journal of Radiology, 51, 1-4.

Fawcitt, R. A., Forbes, W. S. C., Isherwood, I., Morris, A. I., Marsh, M. N., and Turnberg, L. A. (1978). Computed tomographic scanning in liver disease. Clinical Radiology, 29, $251-254$.

Havrilla, T. R., Haaga, J. R., Alfidi, R. J., and Reich, N. E. (1977). Computed tomography and obstructive biliary disease. American Journal of Roentgenology, 128, 765-768.

Kreel, L. (1977). Computerised tomography and the liver. Clinical Radiology, 28, 6, 571-581.

Morris, A. I., Fawcitt, R. A., Marsh, M. N., and Isherwood, I. (1977). Value of whole body computerised axial tomography in the diagnosis of liver disease. Gut, 18, A417.

Stephens, D. H., Sheedy, P. F., Hattery, R. R., and MacCarty, R. L. (1977). Computed tomography of the liver. American Journal of Roentgenology, 128, 579-590.

Vicary, F. R., Cusick, G., Shirley, I. M., and Blackwell, R. J. (1977). Ultrasound and jaundice. Gut, 18, 161-164.

Wiggans, G., Schein, P. S., MacDonald, J. S., Schellinger, D., and Harbertt, J. (1976). Computerised axial tomography for diagnosis of pancreatic cancer. Lancet, 2, 233-235. 